\begin{tabular}{c} 
International Journal of Scientific World, $6(1)(2018) 43-46$ \\
International Journal of Scientific World \\
SPC \\
Website: $\begin{array}{c}\text { www.sciencepubco.com/index.php/IJSW } \\
\text { doi: } 10.14419 / i j s w . v 6 i 1.8558 \\
\text { Research paper }\end{array}$ \\
\hline
\end{tabular}

\title{
Addressing a few misleading concepts in physics
}

\author{
Pramode Ranjan Bhattacharjee * \\ Retired Principal, Kabi Nazrul Mahavidyalaya, Sonamura, Tripura 799131, India \\ *Corresponding author E-mail: drpramode@rediffmail.com
}

\begin{abstract}
This paper is concerned with a few misleading issues which are widespread in scientific literature. With a view to getting rid of the ambiguities present in the traditional literature regarding the said issues, proposed solution to each of those issues has been offered. The author feels that with a view to deepening thought and understanding as well as to upgrade the relevant literature at undergraduate level, it is a high time to consider immediate implementation of the present proposal in regard to the pertinent issues raised in this contribution.
\end{abstract}

Keywords: Angular velocity; Axis of rotation; Gravitational intensity; Gravitational potential; Moment of inertia.

\section{Introduction}

It is well-known that the theoretical as well as the experimental study of physical science is based on a lot of well-established conventions. Every one concerned with the study of physical science is supposed to follow those conventions having International recognition. Unfortunately, it has been detected that wellestablished conventions having no resemblance with the wellknown mathematical laws also prevail in the long-running literature of physical science. Getting rid of those ambiguous conventions with alternative flawless replacements should be given topmost priority for the enhancement of the study of physical science. With that point in mind, the long-used concepts of dimensional formula as well as unit of a derived physical quantity have been revisited in [1] along with considering the traditional procedure employed in solving numerical problems [2], [3] in physics. It has been discovered that the traditional representations of dimensional formula as well as the unit of a derived physical quantity, the long-used procedure of solution of numerical problems in physics are flawed [1]. In order to dispense away with the need of such ambiguous representations and procedure of solution of numerical problems in physics, unambiguous alternatives have been proposed in [1]. It would be worth mentioning here that the development of the aforesaid discoveries of flaw had been accomplished on the basis of the well-known 'laws of indices' in elementary algebra [4] and the following fundamental assertion prevailing in [5]. "For purposes of calculation it is the measure of the magnitude that is of importance, and, to avoid a tedious prolixity of statement, such an expression as "a velocity V" will often be used in the sense "a velocity whose measure is V units of velocity.", We are often to go through statements like: a body of mass ' $\mathrm{m}$ ', a particle moving with velocity ' $\mathrm{v}$ ', a force ' $\mathrm{P}$ ' acting on a particle, and so on. The fundamental question that has been awaiting here is what about ' $\mathrm{m}$ ', ' $\mathrm{v}$ ', ' $\mathrm{P}$ ', etc.? When we say that a body is of mass ' $\mathrm{m}$ ', is it exclusively the numerical value of mass of the body in a particular system of unit? If not, does ' $m$ ' correspond to the numerical value of mass of the body along with its unit in the system of unit under consideration? Same type of questions are also awaiting here regarding ' $\mathrm{v}$ ' and ' $\mathrm{P}$ '.
In view of the fundamental assertion mentioned earlier from [5], it is clear that in the statement "a body of mass ' $m$ '", ' $m$ ' is exclusively the numerical value of mass of the body in a particular system of unit. In a similar manner, ' $\mathrm{v}$ ' is exclusively the numerical value of the velocity of the particle in the system of unit considered and ' $\mathrm{P}$ ' is exclusively the numerical value of the force acting on the particle in a particular system of unit, and so on. But unfortunately, this fundamental fact has been overlooked in solving numerical problems in physics [2], [3]. At the same time no account of the well-established laws on indices of elementary algebra prevailing in [4] has been taken proper care of in deriving the dimensional formula as well as unit of a derived physical quantity. For example, in finding the dimensional formula as well as the unit (say SI unit) of the derived physical quantity 'velocity', we are always to make use of the well-established law ' $\mathrm{a}^{-n}=\frac{1}{\mathrm{a}^{\mathrm{n}}}$ ' to obtain the results as $\mathrm{LT}^{-1}$ and $\mathrm{ms}^{-1}$ respectively. But in doing so, the fundamental fact that in the aforesaid law ' $a^{-n}=\frac{1}{a^{n}}$ ' of indices, ' $a$ ' is a non-zero real number with ' $n$ ' having all rational values, has always been overlooked and since none of ' $L$ ', ' $T$ ', ' $\mathrm{m}$ ', and 's' satisfies these conditions, the application of the said law of indices of elementary algebra to these problems is questionable and an ambiguous one. Thus the procedure of finding dimensional formula and unit of a derived quantity in the literature of physical science has been claimed to be flawed in [1] and relevant unambiguous way out to deal with such cases has also been proposed in [1].

This paper is an extension of [1]. It considers some more misleading issues in Physics/Science which are widespread in the scientific literature. The issues considered in the present study are:

i). The ambiguity resulting from non-specification of the direction of the axis of rotation.

ii). The misleading statement "Moment of inertia of a rigid body changes with the change of the axis of rotation".

iii). The ambiguous concept of considering the axis of rotation as the one perpendicular to the plane of motion of a particle moving round a circle and passing through the centre of the circular track, 
iv). The ambiguous procedure of finding the gravitational intensity at a point lying on the surface of a thin spherical shell found in the traditional literature of Physics.

v). The long-running misleading concept of joining two adjacent plotted points on a graph drawn with experimental data points.

The aforesaid pertinent issues are examined first to find that all of them are misleading and ambiguous. To get rid of the prevailing ambiguities, proposed solution in regard to each of those issues has also been offered.

\section{Ambiguous issues prevailing in the tradi- tional literature of science and getting rid of the same}

It has been reported in [1] that the long-used concepts of dimensional formula as well as the unit of derived physical quantity are not at all in compliance with the well-established laws on indices in elementary algebra [4] and are therefore fundamentally flawed. Flawless replacements of the aforesaid concepts have been offered in [1]. The fundamental assertion quoted in the "Introduction" section from [5] also leads to the discovery of flaw in the usual procedure of solving numerical problems in physics [2], [3]. In this section five more ambiguous issues are considered which are widespread in the traditional literature of Physics/Science. These are:

i). Does the axis of rotation possess a unique direction? If so, why such a direction has not been identified at many places of relevant discussion in the traditional literature?

ii). Is the statement "The moment of inertia of a rigid body changes with the change of the axis of rotation" found in traditional literature [6], [7] a correct one?

iii). Is the traditional definition of angular velocity correct? Will it be defined with respect to only the unique axis passing through the centre of the circle?

iv). Is the procedure of calculation of gravitational intensity at a point on the surface of a thin spherical shell prevailing in existing texts [8], [9], [10], [11] not ambiguous?

v). How far can the joining of two adjacent plotted points on a graph drawn with experimental data points [12], [13] be justified?

The aforesaid five issues are examined below one after another to find that there is existence of ambiguity in each of them and to get rid of the same, relevant suggestions have been proposed.

i). It is, of course, true that the axis of rotation must have a direction. This fact reflected in many places of the traditional literature [14], [15] shows that the axis of rotation must have a unique direction.

The quoted lines from [15] are: "The only fixed, unique direction for a rotating wheel is the axis of rotation, so it is logical to choose this axis direction as the direction of the angular velocity. Left with two choices about direction, it is customary to use the right hand rule to specify the direction of angular quantities." Also the quoted lines from [14] are: "The direction of curl $\mathbf{F}$ points along the axis of rotation, but we need to specify in which direction along this axis the vector should point."

But at the same time many literature [7], [9], [16] exist in which the said fundamental fact has been overlooked and they need immediate correction with incorporation of direction like [14], [15] to the axis of rotation to get rid of the relevant ambiguity.

ii). The statement like "Moment of inertia of a rigid body changes with the change of the axis of rotation" found in many places of the traditional literature [7] and [9] appears to be correct. But strictly speaking it is not so. Considering torsional oscillations of a rigid body about a vertical axis passing through its C. G., we find that after each halfoscillation, there is a change of the axis of rotation (oscillation) because of the change in the direction of rotation of the rigid body. But still the moment of inertia of the body about the axis of oscillation remains the same.
Thus there is a need to rewrite the aforesaid statement in correct form which is: "The moment of inertia of a rigid body will change with the change in position (excluding the collinear unlike parallel position) of the axis of rotation of the rigid body."

iii). As shown in Fig. 1, let a particle move from $A$ to $B$ in 1 second. Then with respect to the axis of rotation (which is normally taken as the axis passing through the centre ' $\mathrm{O}$ ' of the circle and perpendicular to the relevant plane in which the circle lies) the angular velocity of the particle in radian per second is:

$$
\omega_{1}=\frac{\text { Angle } \mathrm{AOB}}{1}=\text { Angle } \mathrm{AOB}=\theta, \text { say. }
$$

Again with respect to an axis of rotation passing through the point $\mathrm{P}$ and perpendicular to the plane in which the circle lies, as shown in Fig. 1, the angular velocity in radian per second is

$$
\omega_{2}=\text { Angle } \mathrm{APB}=\alpha \dot{\alpha} \text {, say. }
$$

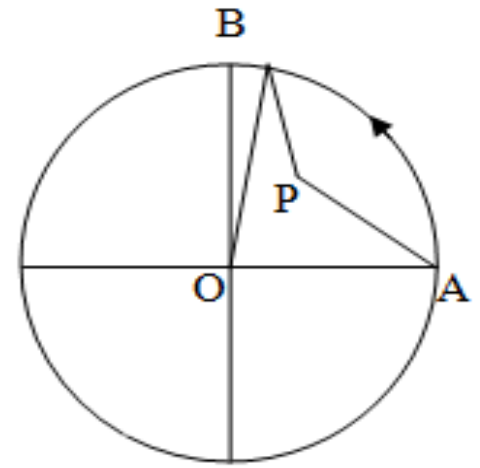

Fig.1 Diagram considered for discussion on angular velocity.

Now, since $\alpha>\theta$, it follows that here $\omega_{2}>\omega_{1}$.

It is because of this reason that a moving train appears to move with more angular velocity with respect to a nearer observer as compared to a distant observer. Again on account of the same reason, to a person in a train moving with uniform angular velocity, nearer object outside the train will appear to move faster than that of a distant object lying outside the train.

It thus appears from above that the traditional concept regarding the definition of angular velocity is ambiguous and needs correction with incorporation of the fact discussed above.

iv). With reference to Fig. 2, where $\mathrm{a}=$ radius of the thin spherical shell, $\mathrm{r}=$ the distance of the point $\mathrm{P}$ from the centre $\mathrm{O}$ of the shell, calculation of gravitational potential at points lying outside, inside, and on the surface of a thin spherical shell as found in [8], [9], [10], [11], give the following results.

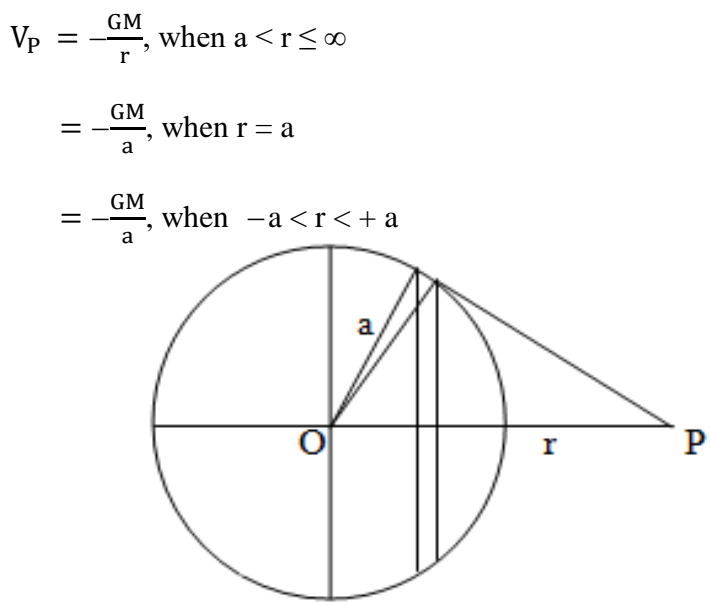

Fig. 2 Diagram for the derivation of gravitational potential and intensity at any point due to a thin spherical shell. 
In the traditional literature [8], [9], [10], [11], the calculation of gravitational intensity making use of the aforesaid expressions for gravitational potential seems to be very much confusing to the case when $r=a$, i.e. when the point lies on the surface of the thin spherical shell.

The alternative approach incorporated by the authors in [8] for the calculation of the gravitational intensity at a point lying on the surface of a thin spherical shell is something like below.

We know that the potential outside the surface of the shell is given by

$$
\mathrm{V}=-\frac{\mathrm{GM}}{\mathrm{r}}
$$

Hence, $f=-\left(\frac{d v}{d r}\right)=\frac{d}{d r}\left(\frac{G M}{r}\right)=-\left(\frac{G M}{r^{2}}\right)$

This value of attraction continues up to the surface of the spherical shell and hence on the surface, the intensity is

$f=-\left(\frac{G M}{a^{2}}\right)[$ Since $r=a$, on the surface $]$

Calculation of gravitational potential and intensity due to a thin spherical shell has also been presented in [11]. The approach for finding the gravitational intensity at a point lying on the surface of a thin spherical shell is as follows.

When $\mathrm{P}$ is on the shell, intensity at $\mathrm{P}$ is

$\mathrm{FP}_{\mathrm{P}}=+\left(\frac{\mathrm{dv}}{\mathrm{dr}}\right)=\frac{\mathrm{d}}{\mathrm{dr}}\left(-\frac{\mathrm{GM}}{\mathrm{r}}\right)=\left(\frac{\mathrm{GM}}{\mathrm{r}^{2}}\right)$

The same type of calculation regarding the derivation of the expression for gravitational intensity at a point lying on the surface of a thin spherical shell also exists in [9], [10]. Now, it may be recalled that each of the above cited traditional literature [8], [9], [10], [11], makes use of the expression for the gravitational potential at a point lying outside the spherical shell (viz. the expression $V_{P}=-\frac{G M}{r}$, when $\left.a<r \leq \infty\right)$ for the calculation of the gravitational intensity at a point lying on the surface of a thin spherical shell (i.e. when $r=a$ ). Strictly speaking this type of calculation of gravitational intensity at a point lying on a thin spherical shell for which $r=a$, by making use of the expression of the potential $V_{P}$, when the point $\mathrm{P}$ lies outside the spherical shell for which " $\mathrm{r}$ " is never equal to " $\mathrm{a}$ " is fundamentally flawed and it deserves special attention.

In view of above, with a view to avoiding any further confusion in the calculation of the gravitational intensity at a point lying on the surface of a thin spherical shell, the following novel approach is being offered.

In this particular case, we have,

$$
\begin{aligned}
& \left(\frac{d V_{P}}{d r}\right)_{r=a}=\left[\operatorname{Lt}_{h \rightarrow 0} \frac{\left[V_{P}(r+h)-V_{P}(r)\right]}{h}\right] r=a \\
& =\mathrm{Lt} \quad \frac{\left[\mathrm{V}_{\mathrm{P}}(\mathrm{a}+\mathrm{h})-\mathrm{V}_{\mathrm{P}}(\mathrm{a})\right]}{\mathrm{h}} \\
& \mathrm{h} \rightarrow 0 \\
& =\underset{h \rightarrow 0}{\operatorname{Lt}} \frac{\left[-\left(\frac{G M}{a+h}\right)-\left(-\frac{G M}{a}\right)\right]}{h} \\
& =\mathrm{Lt} \quad \frac{\left[-\mathrm{GM}\left(\frac{1}{\mathrm{a}+\mathrm{h}}-\frac{1}{\mathrm{a}}\right)\right]}{\mathrm{h}} \\
& \mathrm{h} \rightarrow 0 \\
& =\mathrm{Lt} \quad \frac{[-\mathrm{GM}(-\mathrm{h})]}{\mathrm{h}(\mathrm{a}+\mathrm{h}) \mathrm{a}} \\
& \mathrm{h} \rightarrow 0
\end{aligned}
$$

$$
\begin{aligned}
= & L t \frac{G M}{(a+h) a} \\
& h \rightarrow 0 \\
= & \frac{G M}{a^{2}}
\end{aligned}
$$

Hence Gravitational intensity at $r=a$ is given by,

$\mathrm{E}=\left(-\frac{d V_{P}}{d r}\right)_{r=a}=-\frac{G M}{a^{2}}$

v). While performing experiments in laboratories, we are always in need of collecting experimental data. Thereafter, there is often a need to represent the graphical behavior of the parameters observed as well. In this context, as per guidelines available in standard literature [12], [13], it is always advisable to plot the data collected in a graph paper using proper scales along both the axes with maximum enlargement (i.e. using maximum area of the graph paper) and draw a line/smooth curve so as to pass through as many points as possible. The fundamental question that has been awaiting in the said context is: How far is the joining of two adjacent plotted points on a graph drawn with experimental data points justified from realistic view point?

It would be worth mentioning here that measurement of experimental parameters involved in a study could only be carried out within the limits of the experimental apparatus, i.e. up to the least counts of the instrument/apparatus used in the measurement.

Now, in between any two adjacent points of an experimental graph, there exist infinite number of points, the relevant data corresponding to each of which could not be obtained from experimental observations. So, how is it possible to know the nature of the experimental curve in between two closest experimental points on the graph paper and proceed for drawing a line or a smooth curve so as to pass through as many points as possible as in [12], [13] without having in mind at least the statistical method(s) for curve fitting?

It may be noted that this type of situation never arises in drawing theoretical graphs. This is because in drawing, for example, the theoretical graph corresponding to the relation, $\mathrm{y}=\mathrm{x}$, where $\mathrm{x}$ and $y$ are real continuous variables, any arbitrary points satisfying the relation $\mathrm{y}=\mathrm{x}$ may be selected. Those points may be plotted on a graph paper in the usual way and joined to obtain a straight line. Any two adjacent points on the graph paper in this case may be joined by straight line segment on account of the fact that each of the infinite number of points lying in between any two adjacent points on the graph paper in this case will satisfy the equation, $\mathrm{y}=$ $\mathrm{x}$, and will lie on the corresponding straight line joining those two closest points on the graph paper.

With a view to getting rid of the ambiguous concept of drawing experimental graphs, the following realistic proposal is being offered for those who are not well aware of the statistical method(s) for curve fitting. The plotted experimental data points should never be joined to form a straight line or a curve as has been advised in [12], [13]. On the other hand, such plotted experimental data points should appear on the graph paper in the background of the theoretical graph drawn by making use of the quantitative/theoretical relation, if exists between the two parameters under consideration. Otherwise those plotted experimental data points should always remain isolated on the graph paper and in no circumstances, they should be joined.

\section{Conclusion}

In order to enhance the level of teaching, a teacher is always in need of upgrading himself/herself by gathering updated information on the relevant topic to be taught from various resources. In classroom teaching, he/she is supposed to impart lesson on a particular topic by way of discussion/delivering a lecture. In addition to upgrading himself/herself, it is the essential quality of a 
good teacher to pay simultaneous attention to the clarity of his/her presentation on the topic to be taught in the classroom. In other words, a good teacher always tries to see that his/her lecture delivered on a topic in the classroom always remains absolutely free from any kind of ambiguity. This is essential not only to make his/her lecture in the classroom attractive, but it could then be much clear to the taught leaving no room for confusion and that ultimately results in effective teaching.

For the clarity of presentation which ultimately results in effective teaching, there is an urgent need for the development of precise and unambiguous concepts by getting rid of the ambiguous concepts which are prevailing till today in the traditional literature. Efforts have already been made in such regard and the works [1], [17], [18], [19], [20] deserve special attention.

Questions have already been raised in [1] regarding the validity of the well-established concepts on dimensional formula as well as unit of a derived physical quantity which are widespread in the scientific literature. Furthermore, the traditional procedure of solution of numerical problems in physics [2], [3] has also been examined and reported to be flawed in [1]. Discovery of ambiguity in the traditional definitions of angles of incidence, reflection, and refraction has been reported in [17] along with the development of the definitions of unambiguous angles of incidence, reflection, and refraction, which ultimately leads to the generalized vectorial laws of reflection and refraction. The traditional concept of the scalar nature of plane angle has been found to be ambiguous and is replaced by the unambiguous concept of vectorial angle in [18]. The traditional concept behind the procedure of drawing enlarged experimental curve manually has been reported to be flawed in [19] with simultaneous development of relevant flawless procedure in such regard. The long-running definition of angle of diffraction in Physical optics as well as that of the glancing/grazing angle in Xray crystallography are both reported to be ambiguous in [20] along with simultaneous development of unambiguous definitions of both the aforesaid two angles.

This paper being an extension of [1], examines some of the long running ambiguous issues which are prevailing in the scientific literature. Violation of the fundamental fact that, the axis of rotation must have a direction, in many places of the traditional literature has been considered first. The misleading statement "Moment of inertia of a body changes with the change of the axis of rotation", which is found in many places of traditional literature has been subsequently considered and corrected with alternative unambiguous statement. A fruitful solution to a typical real world problem subsequently dealt with raises a question regarding the long-running concept of unique position of the axis of rotation. The misleading procedure of calculation involved in the traditional literature for finding the expression for the gravitational intensity at a point lying on the surface of a thin spherical shell has been pointed out next and a novel unambiguous sophisticated procedure has been offered for the said purpose. Thereafter the usual procedure of joining adjacent data points in drawing experimental graphs has been examined and found to be questionable at least to those students who do not have the scope to go through the statistical procedure for finding the line of best fit on account of the limitation of the relevant curriculum of their study. For such taught students, the realistic procedure to be adopted has been finally offered.

From the view point of readability and clarity of presentation, it is felt that like the recognition of the flaws reported in [1] with relevant proposed solutions, it is a high time to get rid of the ambiguities reported in this paper with simultaneous acceptance of the solutions proposed. This is essential to spread the message in regard to the ambiguities reported in the present paper to the teaching community so as to upgrade themselves in regard to their quality of teaching.

\section{References}

[1] P. R. Bhattacharjee, Physics standing on baseless platform, The old and new concepts of physics 5(2) (2008) 413-423.
[2] D. Halliday and R. Resnick, Physics for students of Science and Engineering, John Wiley and Sons, INC., New York, 1962.

[3] H. C. Verma, Concepts of Physics, Vol.1, Bharati Bhawan, Patna, India, 1997.

[4] S. N. De, Higher Secondary Mathematics, Vol. 1, Chhaya Prakashani, Kolkata, India, 1998.

[5] G. A. Gibson, An elementary treatise on the calculus, Macmillan and Co., Limited, New York, 1949.

[6] R. Fitzpatrick, 2006, Rigid body rotation, Retrieved on 26 January, 2017

from http://www.farside.ph.utexas.edu/teaching/301/lectures/node99.htm 1.

[7] Moment of Inertia, Retrieved on 26 January, 2017 from: http://www.kwon3d/moi/.com/theory moi.html.

[8] R. Sengupta and H. L. Chatterjee, A treatise on general properties of matter, Vol. I, $7^{\text {th }}$ revised edition, New Central Book Agency, Calcutta, India, 2010

[9] A. B. Gupta, College Physics, Vol. I, Books and Allied (P) Ltd., Calcutta, India, 2001

[10] A. N. Konar, Classical Physics, Vol. 1, Sree Tara Prakasani, Kolkata, India, 2001.

[11] D. B. Sinha and J. M. Das Sharma, College Physics, Vol. I., Modern Book Agency Private Limited, Calcutta, India, 1976.

[12] E. S. Ferry, A Course of Practical Physics for Students of Science and Engineering, Lafayette, Indiana, 1903.

[13] W. William, A Text-Book of Practical Physics, Longmans, Green, and Co., New York, 1913.

[14] The idea of the curl of a vector field - Math insight, Retrieved on 26 January, 2017 from: http://www.mathinsight.org/curl_idea

[15] Vector Properties of Rotational Quantities - HyperPhysics, Retrieved on 26 January, 2017 from: http:// www.hyperphysics.phyastr.gsu.edu/hbase/rotv.html.

[16] Appendix A On the Rotation Axis of the Sun SoHO - MDI, Retrieved on 26 January, 2017 from: http://www.soi.stanford.edu/papers/dissertations/giles/thesis/PDF/a ppendixA.pdf.

[17] P. R. Bhattacharjee, The generalized vectorial laws of reflection and refraction, European Journal of Physics 26(5) (2005) 901-911, .https://doi.org/10.1088/0143-0807/26/5/022.

[18] P. R. Bhattacharjee, Plane angle emerging as a vector quantity, Concepts in pure and applied Science 2 (2015) 1-4.

[19] P. R. Bhattacharjee, Discovering the existence of flaw in the procedure of drawing enlarged experimental curve, Journal of Mathematical Sciences \& Mathematics Education 11(1) (2016) 23-29.

[20] P. R. Bhattacharjee, Discovery of ambiguity in the traditional definitions of angle of diffraction and glancing angle, Optik 130 (2017) 702-707, https://doi.org/10.1016/j.ijleo.2016.10.114. 